\title{
Human Machine Synergies in Intra-Logistics: Creating a Hybrid Network for Research and Technologies
}

\author{
Aswin Karthik Ramachandran Venkatapathy, Haci Bayhan, Felix Zeidler, Michael ten Hompel \\ TU Dortmund University, \\ Lehrstuhl für Förder- und Lagerwesen, \\ Joseph-von-Fraunhofer-Straße 2-4, 44227 Dortmund \\ \{aswinkarthik.ramachandran, haci.bayhan, felix.zeidler, michael.tenhompel\}@tu-dortmund.de
}

\begin{abstract}
The purpose of the article is to outline the futuristic vision of Industry 4.0 in intra-logistics by creating a hybrid network for research and technologies thereby providing a detailed account on the research centre, available technologies and their possibilities for collaboration. Scientific challenges in the field of Industry 4.0 and intra-logistics are identified due to the new form of interaction between humans and machines. This kind of collaboration provides new possibilities of materials handling that can be developed with the support of real-time motion data tracking and virtual reality systems. These services will be provided by a new research centre for flexible humanmachine cooperation networks in Dortmund. By the use of various reference and experiment systems various real-time scenarios can be emulated including digital twin simulation concepts. Big data emerges as an important paradigm in this research project where all systems are made flexible in terms of networking for all the systems to consume the data produced and also to combine all the data to arrive at new insights using concepts from machine learning and deep learning networks. This leads to the challenge of finding a common syntax for inter-operating systems. This paper describes the design and deployment strategies of research centre with the possibilities and the design insights for a futuristic Industry 4.0 material handling facility.
\end{abstract}

\section{INTRODUCTION}

$\mathbf{F}$ OR MANY YEARS, scientists focus on the new change of paradigm in the organisation and management of the whole value-added chain and the impact on the economy and society which are caused by Industry 4.0. With Industry 4.0 , the technologies, services and methods used in industrial production and logistics are changing. Dynamic, real-time and self-organising value-added networks emerge, based on the availability of the relevant information in real-time through the networking of all parties involved in the entire valueadded process and the interconnection of objects, humans and systems [1]

The technological base for the Industry 4.0 is formed by data networked production facilities, products and materials as well as transport technologies, which are equipped with sensors and decentralised IT intelligence. These intelligent cyberphysical systems (CPS), which are connected over the Internet, are able to autonomously organise, control and adapt the sequence of value-added processes and the corresponding logistical functions to external requirements. The current "tech- nology push" in the design and introduction of autonomous CPS-based production systems, advancing digitisation, and automation lead to the development of new forms of services and work organisation [2]. This new forms of services with collaborative machines can be simulated to an extent but can only be understood deeply with a level of trust for adoption into industry when demonstrators are developed.

Thus, the change driven by Industry 4.0 is not predetermined, but can be shaped [2][3]. In addition to the question about the organisation of responsible and goal-oriented action in the human-machine interaction (HMI), there should be a need-oriented debate on the topic of hybrid services. A key factor for the successful transition to Industry 4.0 is the physical implementation of demonstrators and the execution of experiments in a realistic intra-logistics environment. This article describes one such demonstrator where experiments for scenarios of Industry 4.0 can be conducted. The requirements for such a research centre is discussed in section 2 giving a detailed account on conceptual description and the scientific objectives followed by surveying existing research centres in section 3. Section 4 describes all the systems that are deployed in the research centre with information about their flexibility and interoperability. Section 5 concludes the article with the summary of the systems deployed with the direct research goals arising because of the interoperability and collaboration of the systems.

\section{CONCEPTUAL DESCRIPTION AND SCIENTIFIC OBJECTIVE}

In order to cope up with the dynamics and complexity increase and thus not to lose the competitive connection, companies have to increase the adaptability of their processes and business models [4]. It is necessary to generate adaptation measures that combine the advantages of technological innovations as well as human skills. In order to analyse and evaluate the efficiency potential of the emerging sociotechnical systems, in Dortmund an interdisciplinary research project is initiated that is funded by the German Federal Ministry of Education and Research (BMBF) and entitled as "Innovationslabor - Hybride Dienstleistungen in der Logistik" 


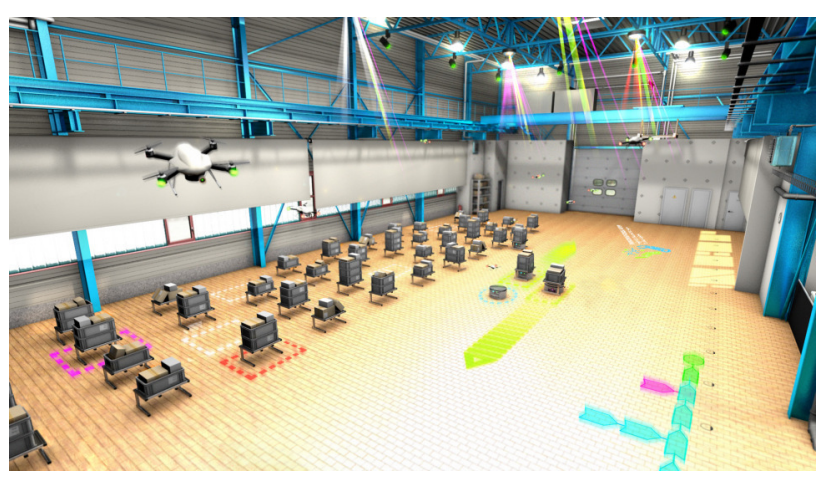

Fig. 1. Structural insight into the research centre

[5]. Decentralisation, networking and localisation form the three basic pillars of an efficient hybrid work environment. With these three pillars a lot of other virtual elements can be included in the research focus such as creating a digital twin [6].

This novel form of an interdisciplinary and cross-process research environment is intended to contribute, amongst others, to answer the following central scientific questions:

- Through the emergence of hybrid cooperation networks $(\mathrm{HCN})$, in which humans and machines support and complement each other in a common work space, the question arises of how responsible, secure and purposive action can be designed and organised in the future HMI.

- On the one hand, the continuously increasing amount and complexity of data in the field of intra-logistics can lead to an additional burden on employees; e.g. in the form of demotivation or excessive demand. On the other hand, the available data can improve, among other things, the quality of employee decisions. Therefore it is essential to answer the question how the innate abilities of employees (such as creativity, motor skills, experience, intuition) can optimally be combined with the abilities of technical (assistance-) systems (e.g. for data evaluation or information visualisation).

- In answer to the research question how technical systems can perceive, analyse and evaluate their environment more intelligently, e.g. by means of big-data analysis or machine intelligence techniques, a contribution is to be made to increase their adaptability and their ability to interact with humans.

- The increasing networking and decentralised control of the entities in a HCN lead to the fact that the amount of data to be processed increases by a factor of 1000 per decade [7]. From this, the question of how the emerging data volume can be transmitted securely, wirelessly and in a system guaranteed time.

For research, to develop scenarios, use cases and experimental situations the research centre is furnished with flexible reference and experiment systems and their interoperability which is described in the following chapters with a survey of research centres that are used for deriving system requirements. A 3D render of the research is shown in fig 1 .

\section{SURVEY OF RESEARCH CENTRES}

This section surveys existing systems in various research domains and to arrive at the system requirements for the research centre. As stated in conceptual description of the research centre, it is important to focus on localisation and navigation systems for the future logistics facilities. From the systems [8], [9], [10], [11], [12], [13], [14], [15], [16] and the publications using these systems, it is clear to use an optical localisation system that is capable to provide location data of objects in real time in 3D. From [12] and [13], it is clear to understand the communication characteristics of various wireless communicating entities is important. Due to the amount of usable data that can be gathered from radio communication and to acquire the data and process this data on the radio system, a radio reference system is proposed in the requirements of the research centre which is capable of multi-standard baseband processing [14]. These reference systems should run synchronised with the ability to acquire data from all the available systems. The requirements from the reference are gathered from these existing research centres and with the experiment systems being generated from a futuristic Industry 4.0 warehouse where a strong HMI takes place with IoT systems playing an important role in coordinating the logistics processes.

\section{REFERENCE AND EXPERIMENT SySTEMS IN THE RESEARCH CENTRE}

\section{A. Reference systems}

1) Optical reference system: The motion tracking system is the north star for the research centre which is a real-time localisation system (RTLS). The RTLS system requires small markers to be attached to the objects that are being tracked and with a very minimum calibration effort, the objects can be tracked using the Tracker software provided by the camera manufacturer. This system is a reference system for providing location of the objects in a precision of less than half a meter guaranteed by the software with a limitation of 1 camera having direct line of sight of at least three markers attached to the object after calibration. There are 38 cameras over an area of 570 sq. $m$ to track and localise all the objects i.e. markers within the area. The software also guarantees a maximum computation time for estimating the location in the 3D space which is less than a second. The software exposes application programming interface (API) that can be used as both query on demand or to have a stream of all the objects that are being tracked. Each tracker can be labelled in the software to get the data labelled accordingly when consumed by another application, this provides context to other interoperating systems.

2) Radio reference system: A network of Software defined radios (SDR) equipped with an array of antennas is used for sensing, tracking and to analyse the wireless communication within the hall. All communicating devices within the range of $\mathrm{DC}$ to $6 \mathrm{GHz}$ can be covered with each SDR. The bandwidth is 
up to $120 \mathrm{MHz}$. Each SDR has a clock synchronisation signal which enables all the SDR to have the same clock frequency that algorithms like time-of-flight or time-difference-of-arrival can be performed in this network of SDRs and also with the experimental systems which have low cost transceivers that are not capable of doing such baseband computation. Since ultralow power, low data rate wireless transceivers do not allow such flexibility, these devices help in analysing, developing and to benchmark various wireless protocols, localisation or proximity algorithms.

3) Laser projection system: A visual system to create virtual objects and to represent temporary markings within the research area is created using a laser projection system. This system is formulated as a augmented reality system with a laser projection system software that can be controlled using a user program to create visual representations by focusing fast moving laser with a distance of 1 to 8 meters. The laser projection system can be used as a guidance system for robots or to simulate augmented reality systems, for example, a traffic visualisation with virtual elements taking part in the simulation with physically moving robots.

4) Virtual reality system: To facilitate concepts such as digital twin, to extend a logistics facility in a physical dimension inside the virtual world and to simulate virtual components in a simulation and to quickly understand the implications of a scenario a virtual reality wall with millimetre precise markings are printed in a roll shutter of dimensions 200 sq. m which can be folded when not required for the experiment. The markings are used as reference markers for the virtual reality device camera to estimate the position, orientation and geometrically represent objects in the virtual world in correspondence to the physical world.

\section{B. Experiment systems}

1) Robot systems: Mobile robot swarms and drone swarms complement to the complexity of diverse machines that will be put to use in an Industry 4.0 materials handling facility. These systems will be used for performing distinct experiments at scale that intrinsically provides a faster way to adapt in the industry. Therefore, industrial systems and research platforms that can mimic a materials handling facility is deployed using mobile racks with robots fitted with lifts that can carry the racks from point $\mathrm{A}$ to point $\mathrm{B}$. These robots also have automatic charging stations which provide another dimension of resource planning into the process with limited number of available stations to charge. Drones are currently used in industry for inventory management and transport of valuable items in terms of money or for the process. Therefore, drones that are programmable used as research platforms are deployed which can perform tasks in coordination with humans and other machines within the facility.

2) LR-WPAN and other wireless networks: Wireless sensor networks (WSN) are a derived terminology of Internet of Things (IoT) which targets a specific kind of IoT devices that are low power, low data rate devices that are used for sensing physical parameters. This has been standardised as low rate wireless personal area network (LR-WPAN) in the IEEE Standard. In a materials handling facility, there has been a lot of penetration of such devices that ease out the complexity of processes by providing functionality. Moreover, the data recorded during these operations are accurate without human error and are available real-time that they could be reliably used for predicting and forecasting the processes they are used in. A wireless sensor network with 550 nodes is deployed under the floor, 1 meter apart, across the research centre. Each sensor node is capable of communicating wirelessly in $868 \mathrm{MHz}$ and $2.4 \mathrm{GHz}$ frequency bandwidth in compliance with IEEE 802.15.4 PHY and MAC [14], [17]. Devices are connected to a bus that is used for programming, providing energy for their operation and to reset them individually. Each of the lines with 15 sensor nodes are connected to a computer that can be reprogrammed.

3) Networked computational system: The future of an Industry 4.0 material handling facility will highly rely on the data because of the autonomy of the elements. It does not only depend on the large amount of data that is produced, but also on the metadata that is being generated which gives context to the data and also the availability of the data within the system. To provide context and to provide the data to other systems as it is available, a networked computer with high bandwidth network controller is used for concurrent network connections.

\section{Systems Interoperability}

The networked computational system is connected to the synchronisation clock signal from the radio reference system. The motion capturing system is also connected to the same clock signal. Since it is not possible for each cameras to synchronise its frames before post-processed in the tracker software, the data streamed from the SDK is timestamped with the system time in ticks of the synchronisation clock. The networked computer will consume all the data using various network communication standards. This data is then available as the latest data set of all existing systems in TCP/IP to be consumed by all other systems within the field. For example, the RTLS system data as latest received by the computer from the tracker will be made available for sensors and robots to be used in their individual planning algorithms.

Since, each system communicates with its own standards, the challenges for such a system lies in finding a common syntax for inter-operating systems which also provides guarantees for the time at which the data is available. Fig 2 . shows all the reference systems and experiment systems that are connected in a network. This challenge also addresses the scenario of autonomous industrial systems collaborating within each other to complete a task or a job in a materials handling facility. A system bus with systems communication paradigms or a middleware software that converts different data sources and communication standards in a harmonised way will be a challenge while implementing the research centre and also in a real-time industrial use case. 


\section{Research centre of the Chair of Materials Handling and Warehousing}

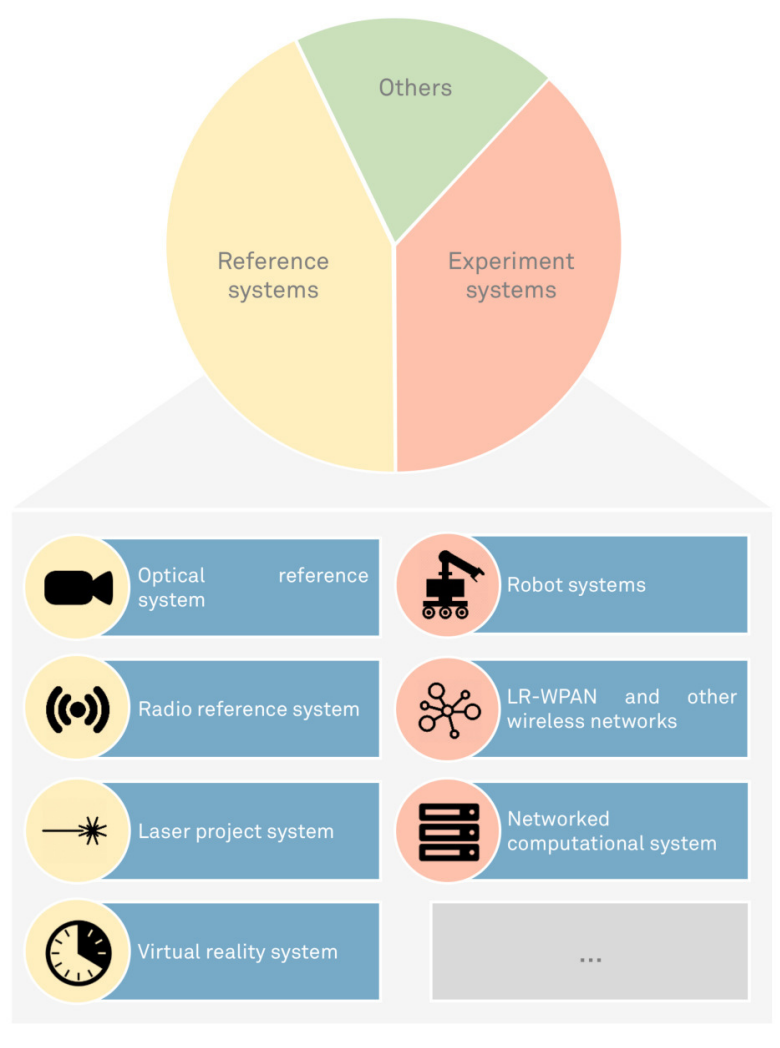

Fig. 2. Inter-operable systems at the Research Centre

\section{Conclusion}

Industry 4.0 and IoT creates new forms of interaction of humans and machines. Based on CPS, a socio-technical work environment is created, in which humans and machines are in dialogue with each other and complete tasks together. The research project "Innovationslabor - Hybride Dienstleistungen in der Logistik" focuses on that new form of HMI. The novel success factor of the research centre is the interdisciplinary collaboration of logistics, IT, engineering, business as well as sociology experts.

The research centre will focus on creating decentralised logistics systems with emphasis on real-time localisation and navigation algorithms. It will also create new areas of development in HMI with emphasis on safety of the workers in the field when collaborating with the machines in the facility.

Through the emergence of HCN in materials handling where humans are supported by machines and machines complement each other in a common work space, this renders a very complex control system for logistics process control. To support in developing such a control system, the research centre is furnished with flexible reference systems and experiment systems.

\section{ACKNOWLEDGMENT}

This article was made in cooperation with the following three projects:

"Innovationslabor - Hybride Dienstleistungen in der Logistik", funded by the German Federal Ministry of Education and Research (BMBF)

"SFB 876, project A4 - Providing Information by ResourceConstrained Analysis", funded by the German Research Foundation (DFG)

"Research Training Group 2193 "Adaption Intelligence of Factories in a Dynamic and Complex Environment", funded by the German Research Foundation (DFG)

\section{REFERENCES}

[1] P. Ittermann and J. Niehaus, "Industrie 4.0 und Wandel von Industriearbeit Überblick über Forschungsstand und Trendbestimmungen," in Digitalisierung industrieller Arbeit, H. Hirsch-Kreinsen, P. Ittermann, and J. Niehaus, Eds. Nomos, 2015, pp. 3253, dOI: 10.5771/9783845263205-32. [Online]. Available: http: //www.nomos-elibrary.de/index.php?doi=10.5771/9783845263205-32

[2] N. Luft, Aufgabenbasierte Flexibilitätsbewertung von Produktionssystemen. Praxiswissen Service, 2013.

[3] H. Hirsch-Kreinsen, "Wirtschafts-und Industriesoziologie," Grundlagen, Fragestellungen, Themenbereiche. München: Juventa, 2005. [Online]. Available: http://www.ulb.tu-darmstadt.de/tocs/207483566.pdf

[4] - "Arbeit 4.0 - der Wandel ist gestaltbar," Jul. 2016. [Online]. Available: https://www.mais.nrw/sites/default/files/asset/ document/arbeit_hirsch-kreinsen_allianz_nrw.pdf

[5] "Innovationslabor I Hybride Dienstleistungen in der Logistik." [Online]. Available: http://www.innovationslabor-logistik.de/

[6] "Adaption Intelligence of Factories in a Dynamic and Complex Environment." [Online]. Available: http://www.grk2193.tu-dortmund. de/en/

[7] M. t. Hompel, C. Kirsch, and T. Kirks, "Zukunftspfade der Logistik, Technologien, Prozesse und Visionen zur vierten industriellen Revolution," in Enterprise -Integration, ser. VDI-Buch, G. Schuh and V. Stich, Eds. Springer Berlin Heidelberg, 2014, pp. 203213, dOI: 10.1007/978-3-642-41891-4_16. [Online]. Available: http: //link.springer.com/chapter/10.1007/978-3-642-41891-4_16

[8] "Kommunikationssysteme." [Online]. Available: http://www.iis. fraunhofer.de/de/ff/kom.html

[9] "Laboratory facilities." [Online]. Available: http://www.es.aau.dk/ sections-labs/Automation-and-Control/Laboratory+facilities/

[10] "Lokalisierung und Vernetzung." [Online]. Available: http://www.iis fraunhofer.de/de/ff/lv.html

[11] "Marker-based motion tracking with Vicon." [Online]. Available: https://www.lfe.mw.tum.de/en/research/methods-and-lab-equipment/ marker-based-motion-tracking-with-vicon/

[12] "Multi Robot Systeme." [Online]. Available: http://www.cyberneum.de/ de/forschungseinrichtungen/trackinglab/multi-robot-systeme.html

[13] "Multisensorische Wahrnehmung und Handlung." [Online]. Available: http://www.cyberneum.de/de/forschungseinrichtungen/trackinglab/ multisensorische-wahrnehmung-und-handlung.html

[14] A. K. Ramachandran Venkatapathy, M. Roidl, A. Riesner, J. Emmerich, and M. t. Hompel, "PhyNetLab: Architecture design of ultra-low power Wireless Sensor Network testbed," in 2015 IEEE 16th International Symposium on A World of Wireless, Mobile and Multimedia Networks (WoWMoM), Jun. 2015, pp. 1-6.

[15] "Startseite I netkops.de." [Online]. Available: http://www.netkops.de/

[16] "TrackingLab." [Online]. Available: http://www.cyberneum.de/de/ forschungseinrichtungen/trackinglab.html

[17] A. K. Ramachandran Venkatapathy, A. Riesner, M. Roidl, J. Emmerich, and M. t. Hompel, "PhyNode: An intelligent, cyber-physical system with energy neutral operation for PhyNetLab," in Smart SysTech 2015; European Conference on Smart Objects, Systems and Technologies, Jul. 2015, pp. 1-8. 